\title{
Strategies for the delay of surgery in the management of resectable hepatobiliary malignancies during the COVID-19 pandemic
}

\author{
S. Bennett MD MSc, ${ }^{*}$ K. Søreide MD PhD, ${ }^{\dagger}$ S. Gholami MD, ${ }^{\ddagger}$ P. Pessaux MD PhD, ${ }^{\S}$ C. Teh MD, \\ E. Segelov MBBS PhD, ${ }^{\#}$ H. Kennecke MD, ${ }^{\ddagger}$ H. Prenen MD PhD, ${ }^{* *}$ S. Myrehaug MD, ${ }^{*}$ D. Callegaro MD, ${ }^{*+\dagger}$ \\ and J. Hallet MD MSc*
}

\begin{abstract}
Objective We aimed to review data about delaying strategies for the management of hepatobiliary cancers requiring surgery during the COVID-19 pandemic.
\end{abstract}

Background Given the CovID-19 pandemic, many jurisdictions, to spare resources, have limited access to operating rooms for elective surgical activity, including cancer, thus forcing deferral or cancellation of cancer surgeries. Surgery for hepatobiliary cancer is high-risk and particularly resource-intensive. Surgeons must critically appraise which patients will benefit most from surgery and which ones have other therapeutic options to delay surgery. Little guidance is currently available about potential delaying strategies for hepatobiliary cancers when surgery is not possible.

Methods An international multidisciplinary panel reviewed the available literature to summarize data relating to standard-of-care surgical management and possible mitigating strategies to be used as a bridge to surgery for colorectal liver metastases, hepatocellular carcinoma, gallbladder cancer, intrahepatic cholangiocarcinoma, and hilar cholangiocarcinoma.

Results Outcomes of surgery during the CovID-19 pandemic are reviewed. Resource requirements are summarized, including logistics and adverse effects profiles for hepatectomy and delaying strategies using systemic, percutaneous and radiation ablative, and liver embolic therapies. For each cancer type, the long-term oncologic outcomes of hepatectomy and the clinical tools that can be used to prognosticate for individual patients are detailed.

Conclusions There are a variety of delaying strategies to consider if availability of operating rooms decreases. This review summarizes available data to provide guidance about possible delaying strategies depending on patient, resource, institution, and systems factors. Multidisciplinary team discussions should be leveraged to consider patient- and tumour-specific information for each individual case.

Key Words CovID-19, coronavirus, hepatobiliary cancer, HCC, metastases, cholangiocarcinoma, gallbladder carcinoma

\section{INTRODUCTION}

The evolving Covid-19 pandemic is placing unprecedented pressures on health systems. Many jurisdictions have cancelled or delayed elective surgical activity to spare precious hospital, critical care, and personal protective equipment resources; to increase system capacity; and to limit nosocomial spread of CoviD-191-4. This sudden scale-back in operative resources has led to re-triaging and prioritization of elective surgeries, including cancer cases. As COVID-19 pressures on local resources mount, operative case categorization changes, and criteria become more stringent, resulting in a higher number of cancer surgeries potentially being deferred ${ }^{5}$.

Delays in cancer surgery could risk losing a window for resection, compromising curative-intent surgery, or delaying palliation, with impacts that can vary depending on the timing of the delay in the cancer care continuum. 
Furthermore, as hospital resources-including criticalcare and specialized staff_-become less available, perioperative outcomes could worsen. The ability to progress patients through the usual postoperative care pathways and to rescue potential complications could be compromised. In the mode of "safety first," that compromised ability shifts the risk-benefit balance of surgery for individual patients and diseases, making changes to the usual decisionmaking and triaging for surgical cancer care inevitable and necessary.

Governing bodies and surgical societies have released statements about the need to delay and prioritize elective surgeries, including those for cancer ${ }^{5,6}$. However, those high-level statements lack either detailed disease- and patient-specific guidance or the data and resource information to support decision-making (Table I) ${ }^{7-9}$.

The logistics of care and operating room availability can shift quickly as the pandemic evolves, and surgical decisions have to rapidly adapt. Multiple factors to consider for each case include not just the usual disease and patient factors (chance for cure or further spread of the cancer, risk for complications), but also, particularly in the pandemic, hospital resource use [for example, the need for an intensive care unit (ICU) stay], the likelihood of prolonged hospitalization, and risk of exposure to and outcomes of COVID-19. Weighing all those considerations requires an understanding of expectations with standard-of-care surgery, available mitigating or delaying strategies and their outcomes, and the risks posed by COVID-19 infection.

The aim of the present review was to provide detailed information to support decision-making for the surgical management of hepatobiliary cancers that can be adapted to rapidly evolving situations and surgical resource availability. Rather than developing guidelines or recommendations that could quickly become obsolete, we aimed to summarize within a single review the data to support difficult discussions and decisions. We focus on surgically treatable intrahepatic malignancies and endorse the logistics of operating room workflow and protocols during the pandemic that have previously been outlined ${ }^{3,10,11}$.

\section{METHODS}

A non-systematic search was conducted of the literature (as of 10 April 2020), surgical and oncology society guidelines, and expert statements about the management of the most common hepatobiliary malignancies in surgical oncology practice:

\section{Colorectal liver metastases (CRLMs) \\ Hepatocellular carcinoma (HCC) \\ Gallbladder carcinoma (GBC) \\ Intrahepatic cholangiocarcinoma (ICC) \\ Hilar cholangiocarcinoma}

Relevant data about standard-of-care surgical management and possible mitigating strategies to be used as a bridge to surgery were extracted and summarized. The components of care summarized for each treatment strategy were: short-term outcomes (morbidity and mortality), long-term oncologic outcomes (survival), factors influencing and tools predicting outcomes, and resource requirements for therapy delivery. When available, we also abstracted data about patient outcomes with delays in surgery.

The search and data abstraction were performed by 2 authors (SB, JH), with subsequent review by a multidisciplinary (surgical, medical, and radiation oncology), international (North America, Australasia, and Europe) group of oncologists to ensure applicability within various health systems and in countries at different phases of the current pandemic. No formal consensus criteria were applied to the review.

\section{RESULTS}

\section{Risk of COVID-19 for the Surgical Oncology Patient}

Data about outcomes of COVID-19-infected cancer patients are limited, particularly with respect to surgical oncology. Repeated visits to the hospital, risk from social and nosocomial contacts, and the immunosuppressive effects of surgical stress could contribute to a higher risk of developing COVID-19 for patients with cancer ${ }^{12,13}$. However, that risk is highly confounded by the prevalence of asymptomatic COVID-19 in the community and the lag between infection and symptoms. Information about vulnerability to COVID-19 can guide decision-making for surgical cancer patients. Comorbidities associated with higher risk from COVID-19 (for example, hypertension, diabetes, pulmonary disease, cardiovascular disease) and age could be used to arrive at the most optimal risk-benefit balance ${ }^{14}$.

Two major epidemiologic studies from China drew data from 18 and 10 cancer patients infected with COVID-19 and suggested a higher rate of ICU admission, need for ventilation, and death (39\%) in that patient population compared with the general population $(8 \%)^{12,13}$. The case fatality rate for all cancer patients with COVID-19 infection is reported to be higher than the overall case fatality rate (5.6\% vs. $2.3 \%$ ), although no details are available about that subpopulation ${ }^{15}$. However, most Chinese patients with cancer and COVID-19 in those series were 4 years or more from their initial cancer diagnosis, and only 1 had undergone surgery and 3 had received chemotherapy within a month of COVID-19 infection ${ }^{12}$. A Chinese series of hospitalized patients with COVID-19 that included 105 patients with and 233 without cancer observed that patients with cancer experienced higher rates of severe events of ICU admission, mechanical ventilation, and death ${ }^{16}$. After adjusting for age, sex, and comorbidities, cancer was not independently associated with increased odds of ICU admission (odds ratio: 1.5; 95\% confidence interval: 0.70 to 3.1 ) or death (odds ratio: 2.2; $95 \%$ confidence interval: 0.46 to 5.2 ). Overall, those data cannot definitively support the idea that delaying therapy would protect from COVID-19 infection. However, COVID-19 infection manifesting during the postoperative period is associated with poor outcomes. An early publication described 34 patients who were operated on while incubating COVID-19 that manifested clinically after surgery; all developed pneumonia, $44 \%$ required ICU admission, and $20 \%$ died $^{17}$. A large multicentre cohort study at 235 hospitals in 24 countries included 1128 patients who underwent any operation and were diagnosed with COVID-19 within a 
TABLE I Summary of statements from major surgical and oncology societies about cancer surgery and hepatobiliary malignancy during the COVID-19 pandemic

Society
American Society of Clinical Oncology
- Individual determinations about the need for cancer surgery based on potential harms of delaying surgery, including consideration of the
need for postoperative ICU care.
- Reasonable to consider neoadjuvant therapy or delay in surgery in situations in which neoadjuvant therapy is available, but not
routinely considered:
- Weigh risks of delay in surgery against burden on hospital resources and patient risk of exposure to COVID-19, and
Hepatobiliary cancers specifically: links to statement from Society of Surgical Oncology
For details: https://www.asco.org/asco-coronavirus-information
National Comprehensive Cancer Network
- Cancer surgery is not considered elective, but requires prioritization.
- Shared decision-making optimized with surgeon-to-patient discussions (telephone calls).
Hepatobiliary cancers specifically: no statement
For details: https://www.nccn.org/covid-19/

American College of Surgeons

- Patients should receive appropriate and timely surgical care, including operative management, based on sound surgical judgment and availability of resources.

- Virtual multidisciplinary discussions for triage of cases based on local resources, COVID-19 prevalence, and alternative nonsurgical therapies.

Three phases guide decision-making relative to level of the pandemic:

- Semi-urgent setting - preparation phase

- Urgent setting

- Local resource scarcity

Hepatobiliary cancers specifically: For oligometastatic colorectal cancer, use effective systemic therapy, if available. No statement for hepatocellular carcinoma, intrahepatic cholangiocarcinoma, and gallbladder cancer.

For details: https://www.facs.org/covid-19/clinical-guidance/elective-case

Society of Surgical Oncology

Follows the American College of Surgeons phases of the pandemic.

Hepatobiliary cancers specifically:

Phase 1 - Semi-urgent setting

Procedures to be done:

- Intrahepatic cholangiocarcinoma (symptoms or not)

- Colorectal liver metastases finishing neoadjuvant therapy in which further chemotherapy would be detrimental to liver function

Consider alternative therapies:

- Large intrahepatic cholangiocarcinoma requiring major hepatecomy chemotherapy

- Hepatocellular carcinoma - ablation or liver-directed therapies

- Incidental gallbladder cancer requiring staging or re-resection - delay

\section{Phase 2 - Urgent setting}

Procedures to be done:

- Advanced tumour at risk of becoming unresectable with delay

- Management of complications if interventional approach not feasible

- Bleeding tumours that cannot be managed with interventional radiology, radiation, or endoscopy

Consider alternative strategies:

- Chemotherapy upfront for tumours in which it is not routine, if can be done safely

- Radiation therapy upfront for tumours in which it is not routine, if can be done safely

- SBRT for liver metastasis

- Liver-directed therapy as bridge to surgery
Phase 3 - Local resource scarcity

Procedures to be done:

- Management of complications if interventional approach not feasible

- Bleeding tumours that cannot be managed with interventional radiology, radiation, or endoscopy

Alternative strategies:

- Same as Phase 2

For details: https://www.surgonc.org/resources/covid-19-resources/

European Society of Surgical Oncology

Liaise with colleagues regarding feasibility and practicality of chemotherapy, radiation therapy, and targeted treatment to reduce impact on hospital beds for surgery, and decision on case-by-case basis.

Hepatobiliary cancers specifically: no statement

For details: https://www.essoweb.org/news/esso-statement-covid-19/ 
TABLE I Continued

\begin{tabular}{|c|c|}
\hline Society & Statement \\
\hline \multicolumn{2}{|l|}{ Americas Hepato-Pancreato-Biliary Association } \\
\hline \multicolumn{2}{|c|}{ Follows the American College of Surgeons phases of the pandemic. } \\
\hline \multicolumn{2}{|c|}{$\begin{array}{l}\text { Hepatobiliary cancers specifically: } \\
\text { - Consider patient comorbidities and age to assess relative risks and benefits with potential exposure to COVID-19 compared with } \\
\text { alternative treatment options. } \\
\text { - Consider changes in resources available at various stages of the pandemic. } \\
\text { - For patients facing a potentially prolonged hospital stay or at higher risk for complications requiring ICU management, surgery should be } \\
\text { timed to available resources. }\end{array}$} \\
\hline $\begin{array}{l}\text { Phase 1 - Semi-urgent setting } \\
\text { - Hepatocellular carcinoma - hepatectomy, } \\
\text { transplant, ablation } \\
\text { - Colorectal liver metastases - hepatectomy } \\
\text { for intermediate-acuity surgery and } \\
\text { healthy patient, chemotherapy for } \\
\text { intermediate-acuity surgery and } \\
\text { unhealthy patient } \\
\text { - Intrahepatic cholangiocarcinoma - } \\
\text { hepatectomy for intermediate-acuity } \\
\text { surgery and healthy patient, chemotherapy } \\
\text { for intermediate-acuity surgery and } \\
\text { unhealthy patient } \\
\text { - Hilar cholangiocarcinoma - resection or } \\
\text { transplantation as indicated }\end{array}$ & $\begin{array}{l}\text { Phase } \mathbf{2} \text { - Urgent setting | Phase } \mathbf{3} \text { - Local resources scarcity } \\
\text { - Hepatocellular carcinoma - delay definitive therapy; TACE ablation or observation } \\
\text { - Colorectal liver metastases - chemotherapy } \\
\text { - Intrahepatic cholangiocarcinoma - chemotherapy or embolic therapy } \\
\text { - Hilar cholangiocarcinoma - chemotherapy, chemoradiation, or transfer to a facility with } \\
\text { more resources }\end{array}$ \\
\hline For details: https://www.sages.org/sages-ahpb & 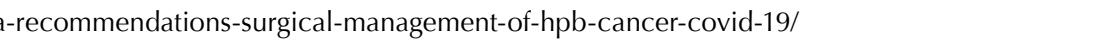 \\
\hline
\end{tabular}

$\mathrm{ICU}=$ intensive care $\mathrm{unit}$; SBRT = stereotactic body radiation therapy; TACE = transarterial chemoembolization.

window of 7 days preoperatively to 30 days postoperative$\mathrm{ly}^{18}$. The overall 30 -day mortality rate was $23.8 \%$, and the rate of pulmonary complications was $51.2 \%$.

\section{Standard of Care and Role of the Multidisciplinary Team}

Despite the limitations imposed by the COVID-19 pandemic, surgeons should strive to provide standard-of-care therapy. All treatment decisions, particularly those in which standard therapy has to be altered or delayed, must be made in a multidisciplinary team. Given that access to chemotherapy and radiation therapies might also be restricted, ongoing communication with oncology colleagues is essential. Treatment options, risks and benefits, and the perceived outcome of each intervention have to be discussed with the patient to arrive at the situational best solution and at informed consent with the patient. Notably, patients and surgeons will have different personalities, values, and expectations, all of which could affect choice of treatment. However, opting for risk-averse pathways might also be justified for the common good in a situation in which resources and access to intensive care support might be restricted.

\section{Therapeutic Options and Resource Use}

Table II summarizes general resource use for possible treatment strategies, to be interpreted in light of specific institutional care processes and resource availability before, during, and after the pandemic.

Hepatectomies are a heterogeneous group of procedures, ranging from laparoscopic wedge resection of a single segment to trisegmentectomy with bile duct resection and reconstruction ${ }^{19}$. Patients at highest risk of morbidity and mortality after hepatectomy are those with comorbidities, an age of 75 years or greater, or underlying cirrhosis; those undergoing major liver resection ( $\geq 4$ liver segments), synchronous colorectal and liver resection, and biliary reconstruction; and those needing blood transfusions ${ }^{19-21}$. The risk of requiring care in an ICU after surgery is higher for patients undergoing major hepatectomy and those receiving blood transfusions ${ }^{22}$. For patients with minor liver disease, a laparoscopic approach can be beneficial, with lower morbidity and a shorter hospital stay ${ }^{19}$.

The risk of morbidity and mortality can be predicted using the National Surgical Quality Improvement Program developed by the American College of Surgeons based on multi-institutional prospectively validated data ${ }^{23}$. Blood transfusion is another important marker of postoperative morbidity and higher use of resources after hepatectomy, and can be estimated using the validated transfusion risk score based on data about preoperative anemia, background liver disease, and extent of planned hepatecto$\mathrm{my}^{24,25}$. Transfusion is especially important to consider, given that the COVID-19 pandemic can lead to allogeneic blood shortages as a result of physical distancing and confinement policies ${ }^{26}$.

Chemotherapy is delivered in an outpatient setting. It involves repeated visits to cancer clinics, with associated risks related to social exposure because of travel, waiting room crowding, and sequential use of a chemotherapy chair that could increase the risk of exposure to COVID-19 infection. Moreover, chemotherapy means exposure to agents that might compromise the quality of the liver remnant and create a risk of immunosuppression ${ }^{27}$.

Percutaneous ablation, such as radiofrequency ablation (RFA), can be delivered as outpatient care in radiology 
TABLE II Safety profile and resource use for treatment strategies

\begin{tabular}{|c|c|c|c|c|c|c|}
\hline Strategy & $\begin{array}{c}\text { Safety profile } \\
\text { (adverse effects) }\end{array}$ & $\begin{array}{l}\text { Clinical } \\
\text { setting }\end{array}$ & Length of stay & $\begin{array}{c}\text { Unplanned } \\
\text { ICU admission }\end{array}$ & $\begin{array}{c}\text { Typical } \\
\text { PPEb }^{b}\end{array}$ & $\begin{array}{l}\text { Immuno- } \\
\text { suppression }\end{array}$ \\
\hline Hepatectomy & $\begin{array}{c}\text { Major morbidity: } 20 \% \\
\text { Mortality: } 2 \%\end{array}$ & Inpatient & 3-6 Days & $5 \%-10 \%$ & Yes & $\begin{array}{l}\text { Relative: } \\
\text { postoperative } \\
\text { SIRS }\end{array}$ \\
\hline Systemic therapy & $\begin{array}{l}\text { Depends on regimen. } \\
\text { Consider neutropenia } \\
\text { and lymphopenia. }\end{array}$ & Outpatient & $\begin{array}{l}\text { Recurrent visits } \\
\text { (every 1-3 weeks) }\end{array}$ & Rare & Yes & Yes \\
\hline \multirow{2}{*}{$\begin{array}{l}\text { Percutaneous } \\
\text { ablation }\end{array}$} & \multirow[t]{2}{*}{$4 \%$ Grade 3 events } & Outpatient & 1 Day & \multirow[t]{2}{*}{ Rare } & \multirow[t]{2}{*}{ Yes } & \multirow[t]{2}{*}{ No } \\
\hline & & $\begin{array}{c}\text { Inpatient } \\
\text { (possible OR) }\end{array}$ & 1-2 Days & & & \\
\hline $\begin{array}{r}\text { Ablative radiation } \\
\text { therapy (SBRT) }\end{array}$ & $\begin{array}{c}6 \%-7 \% \text { Grade } 3 \\
\text { gastrointestinal events } \\
\text { (if proximity to } \\
\text { duodenum or stomach) }\end{array}$ & Outpatient & 3-5 Daily visits & Rare & No & No \\
\hline \multirow[t]{2}{*}{$\begin{array}{l}\text { Intra-arterial liver } \\
\text { embolization }\end{array}$} & \multirow[t]{2}{*}{$\begin{array}{l}15 \%-30 \% \text { Pain, } \\
\text { fatigue, vomiting }\end{array}$} & Outpatient & $\begin{array}{c}1 \text { Day } \\
\text { (might require repeats, } \\
4-6 \text { weeks apart) }\end{array}$ & \multirow[t]{2}{*}{ Rare } & Yes & \multirow[t]{2}{*}{ No } \\
\hline & & $\begin{array}{c}\text { Inpatient } \\
\text { (possible OR) }\end{array}$ & 1-2 Days & & & \\
\hline
\end{tabular}

a Excludes routine planned postoperative admissions for monitoring.

b That is, mask, gown, gloves_PPE requirements for usual care (could vary during COVID-19 pandemic, depending on institutional protocols). $\mathrm{ICU}=$ intensive care unit; $\mathrm{PPE}=$ personal protective equipment; SIRS = systemic inflammatory response syndrome; OR = operating room; SBRT = stereotactic body radiation therapy.

suites or in the operating room, depending on the institution. Grade 3 adverse events in percutaneous ablation occur in $4 \%-5 \%$ of patients ${ }^{28}$. Ablative radiation therapy [stereotactic body radiation therapy (SBRT)] is an outpatient procedure in radiation therapy facilities. The adverse effects profile of SBRT is minimal, with a $6 \%-7 \%$ risk of grade 3 gastrointestinal toxicity ${ }^{29,30}$.

Finally, liver embolization is typically performed in the radiology suite or in the operating room, as an outpatient or inpatient procedure, depending on the institution. Post-embolization syndrome-with fever, abdominal pain, and elevated transaminases-is the most frequent complication $^{31}$. For transarterial chemoembolization (TACE, using drug-eluting beads containing irinotecan) in patients with CRLMs, $15 \%-30 \%$ experience either pain, vomiting, fatigue, or fever, with most adverse events being grade $2^{32,33}$. For TACE in patients with HCC, most adverse effects are also grade 2 . Across studies, $20 \%-30 \%$ of patients experience either pain, fever, nausea, or fatigue, and $5 \%-30 \%$ can develop ascites depending on liver function ${ }^{31}$.

\section{Colorectal Liver Metastases Hepatectomy and Oncologic Outcomes}

The 5 -year overall survival (Os) for patients with resectable CRLMs (liver only) can reach $60 \%$, with a median os of 74 months after curative-intent surgery ${ }^{34,35}$. In a retrospective series of patients with fewer than 4 metachronous metastases treated between 2000 and 2010, longer time from diagnosis to CRLM resection was associated with worse $\mathrm{OS}^{36}$. Median os was 76 months in patients who underwent surgery within 3 months of diagnosis, compared with 58 months for those whose surgery occurred later, adjusted for preoperative receipt of chemotherapy.

\section{Systemic Therapy}

Median survival with systemic therapy alone for unresectable CRLMs has been reported to be 22 months for patients who do not achieve conversion to resectability ${ }^{37}$; other series have reported durations up to 40 months ${ }^{38}$. The availability of effective systemic therapies has led to an increased use of neoadjuvant chemotherapy. In patients with resectable liver metastases (low disease burden), upfront surgery is often favoured. However, for patients with larger lesions or borderline resectable disease, neoadjuvant chemotherapy is the preferred approach. In the neoadjuvant setting, chemotherapy can be used to assess disease biology and patient selection, with potential benefit in recurrence-free survival, but no proven gain in os $^{39,40}$.

Recent guidance from the American College of Surgeons (24 March 2020) recommends that surgery be delayed for patients with oligometastatic colorectal cancer for which effective systemic therapy is available ${ }^{41}$. If case prioritization requires a delay in resection of CRLMs, administration of chemotherapy before resection would be reasonable. For patients who are finishing pre-hepatectomy chemotherapy initiated before an operating room resource restriction, consideration can be given to continuing with further cycles depending on patient tolerance and the balance between risk of hepatotoxicity from chemotherapy (given the expected future liver remnant) and the risk for disappearing metastases that will complicate future curative-intent resection.

\section{Ablation}

Data about the outcomes of percutaneous ablation come from cohorts of patients with unresectable CRLMs. In a phase II randomized trial involving 119 patients with 
unresectable, liver-only CRLMs, better median os was observed for RFA combined with systemic therapy (45.6 months) than for systemic therapy alone $(40.5 \text { months })^{38}$. In retrospective studies of resectable disease, risk of local recurrence is higher and os is lower with ablative techniques than with resection ${ }^{42-44}$. Local recurrence and 5-year os after ablation are reported at $37 \%$ and $27 \%$ respectively for isolated CRLMs ${ }^{42}$. As evidenced by an ongoing randomized noninferiority trial comparing hepatectomy with ablation for resectable liver metastases, percutaneous ablation is considered by some to be a potential curative option for CRLMs ${ }^{45}$.

Retrospective series of patients with unresectable liver metastases treated with SBRT have shown promising disease control. Local control rates at 2 years range from $57 \%$ to $100 \%$ in patients with poor prognosis and unresectable disease $^{46}$. In a phase II trial comparing SBRT plus chemotherapy with chemotherapy alone for oligometastases (13\% liver), median os was prolonged at 41 months for the SBRT regimen compared with 28 months for chemotherapy alone. Compared with percutaneous ablation, SBRT can treat larger lesions, but might be limited for CRLMs localized near the stomach or duodenum ${ }^{29,30}$. Treatment intent with SBRT is not curative; if used as a mitigating strategy, liver resection would subsequently be required. Surgery after SBRT is feasible, although not well described ${ }^{47}$.

If there is no access to the operating room or if pursuing chemotherapy is not possible (or both), ablation could be a delaying strategy to ensure local control while awaiting surgery. Curative-intent treatment requires subsequent resection of the ablated lesion when operating resources are available. The choice for ablation also depends on its technical limitations (tumour size, number, and localization) and required resources depending on institutional protocols (inpatient vs. outpatient care; operating room).

\section{TACE}

Another approach for patients with unresectable or medically inoperable disease is TACE, with a goal of local control and improved os. No prospective comparison of TACE with resection has been conducted. In the unresectable setting, TACE using drug-eluting beads containing irinotecan has an overall radiologic response rate between $60 \%$ and $80 \%$, and a median os ranging from 14 to 25 months ${ }^{33,48}$.

\section{Clinical Risk Tools}

Multiple patient and disease factors influence oncologic outcomes after hepatectomy for CRLMs; clinical risk tools can assist in estimating outcomes if necessary for decisionmaking ${ }^{49}$. The most common tool is the Clinical Risk Score $^{50,51}$. Rated from 0 to 5 , the Clinical Risk Score assigns 1 point for each of these factors: node-positive primary tumour, liver metastases larger than $5 \mathrm{~cm}$, more than 1 lesion, serum carcinoembryonic antigen greater than $200 \mathrm{ng} / \mathrm{mL}$, and a disease-free interval less than 12 months. A score of 0 corresponds to a 5 -year os of $60 \%$, and a score of 5, to a 5-year os of $14 \%$. The Genetic and Morphological Evaluation score is a more recent tool that incorporates molecular information and has better discrimination than the Clinical Risk Score ${ }^{52}$. Six preoperative factors produce a total score in the range $0-7$ : KRAS mutation (1 point), serum carcinoembryonic antigen greater than $20 \mathrm{ng} / \mathrm{mL}$ (1 point), node-positive primary tumour (1 point), tumour burden score in the 3-8 range ( 1 point) or 9 and higher ( 2 points), and extrahepatic disease ( 2 points). The 5 -year os estimate is $73.4 \%$ for patients at low risk (score: $0-1$ ), $50.6 \%$ for those at medium risk (score: $2-3$ ), and $11 \%$ for those at high risk (score: $\geq 4$ ).

\section{Hepatocellular Carcinoma Hepatectomy and Oncologic Outcomes}

Patients with Barcelona Clinic Liver Cancer (BCLC) stage 0 or A HCC are considered for hepatectomy, with some surgeons considering resection for those with BCLC B disease ${ }^{53,54}$. With hepatectomy, these are the estimates for 5 -year os $^{55}$ :

for BCLC $0: 86 \%$.

for BCLC A with 1 lesion of 2-5 cm or 2-3 lesions less than $3 \mathrm{~cm}: 69 \%$.

for BCLC A with 1 lesion of $5 \mathrm{~cm}$ or larger: $57 \%$.

for BCLC B with 2-3 lesions of $3 \mathrm{~cm}$ or larger, or with 4 or more lesions: $50 \%$.

In the present review, we focus on patients who are candidates for resection and who might be delayed or reprioritized during the pandemic. Liver transplantation also plays a crucial role in the management of HCC. Transplantation presents unique considerations related to operating room resource availability, changes in the donor pool, risk for living donors, and risk of infection and transmission of COVID-19, which are beyond the scope of the present review and have been summarized elsewhere ${ }^{56-58}$. Any consideration of strategies meant to mitigate operating room delays for resection of HCC should include the understanding that access to transplantation might still be compromised after the pandemic, such that delaying strategies should not rely on availability of salvage transplantation.

\section{Ablation}

Radiofrequency ablation is associated with local recurrence rates of $0.9 \%, 1.4 \%$, and $25 \%$ for HCCs of less than $2 \mathrm{~cm}$, less than $3 \mathrm{~cm}$, and $3-5 \mathrm{~cm}$ respectively ${ }^{59,60}$. Although no difference in os has been observed with liver resection or RFA for HCCs less than $5 \mathrm{~cm}$ in randomized controlled trials, the local recurrence rate is higher with RFA (5-year recurrence-free survival: $51.3 \%$ vs. $28.7 \%$; hazard ratio: 0.56 ; $95 \%$ confidence interval: 0.40 to 0.78$)^{61}$. If ablation is used without subsequent resection, and if the tumour recurs, patients can be candidates for salvage resection or salvage transplantation with outcomes similar to those with upfront transplantation ${ }^{62}$.

The SBRT modality is used for unresectable HCC, but has not been prospectively compared with resection. Prospective series have demonstrated local control rates between $80 \%$ and $100 \%$ at $1-3$ years ${ }^{63-65}$. The ability to safely deliver SBRT is restricted by the underlying liver function, with a Child-Pugh score of 8 or greater being a relative contraindication ${ }^{66}$. The modality can be a bridge to liver transplantation comparable to both TACE and $\mathrm{RFA}^{67}$.

\section{TACE}

Outcomes of TACE have been reported mostly for unresectable HCC, with the 5 -year os being $30 \%{ }^{68}$. Literature 
about neoadjuvant TACE for HCC reported no difference in Os between TACE followed by hepatectomy and hepatectomy alone in higher-risk HCC ${ }^{69}$. If TACE is used as a strategy to delay surgery, resection should eventually follow so as not to compromise os. Although operating time is longer after TACE, postoperative morbidity and mortality are unchanged ${ }^{69,70}$.

\section{Systemic Therapy}

Sorafenib and lenvatinib are used for advanced and unresectable HCC, with a median os of 12-13 months being reported in the REFLECT trial ${ }^{71-73}$. The emergence of new data showing responses to lenvatinib as evidenced by the Response Evaluation Criteria in Solid Tumors could herald the use of systemic therapy to downstage or delay surgery until optimal conditions are achieved in patients who might not be eligible for other delaying strategies as described earlier.

\section{Clinical Risk Tools}

A variety of scoring tools are available to assess outcomes of liver transplantation for HCC, but fewer are available for hepatectomy. A risk score developed using the Surveillance, Epidemiology, and End Results program database incorporates sex, tumour size, number of tumours, presence of bilobar disease, and major vascular invasion to predict os, but has not been externally validated ${ }^{74}$. The predicted 5 -year os is $69 \%$ with a low-risk score, $51 \%$ with a mediumrisk score, and $19 \%$ with a high-risk score.

\section{Gallbladder Cancer}

\section{Hepatectomy and Oncologic Outcomes}

Gallbladder cancer amenable to resection most often presents incidentally after simple cholecystectomy ${ }^{75,76}$. After a negative metastatic work-up, indications for radical re-resection include $\mathrm{T} 1 \mathrm{~b}$ or higher node-positive disease or a positive cystic duct margin ${ }^{77}$. Resected localized GBC (stages I-II) typically has a 5-year os of $50 \%-60 \%{ }^{78,79}$. Compared with simple cholecystectomy, radical resection with extended cholecystectomy is associated with improved Os for $\mathrm{T} 2$ and higher disease ${ }^{78}$. In T1b tumours, radical resection has been associated with a decrease in the risk of local recurrence without improvement in $\mathrm{os}^{80}$. Major liver resection, including multivisceral resection, is advocated by some for os benefit, but in the setting of the pandemic, the associated increased risks of major morbidity, prolonged ICU stay, and mortality should be carefully weighed against that benefit ${ }^{81,82}$. Finally, it should be mentioned that the extent of hepatectomy and lymph node dissection is debated and heterogeneous between studies, and that available data are biased by selection of fitter patients for extended resection ${ }^{83-86}$.

\section{Delaying Strategies}

For incidental GBC eligible for radical re-resection, delaying strategies can include observation with short-term re-imaging or chemotherapy ${ }^{76}$. Use of chemotherapy is contingent on the availability of an effective regimen for GBC, which can be appreciated based on data from the adjuvant setting. Although the BILCAP trial's intention-to-treat analysis was negative for its primary endpoint, the trial (which included all biliary tract cancers) indicated favourable os with 8 cycles ( 24 weeks) of capecitabine as adjuvant therapy in the per-protocol population (which excluded ineligible patients and patients failing to complete at least 1 cycle of capecitabine) ${ }^{87}$. Although statistical significance was not reached in BILCAP's intention-to-treat population, a survival benefit of several months for those patients with usually dismal outcomes can be considered clinically meaningful. Therefore, when tumour control is needed while surgery is delayed, chemotherapy could be considered $^{88}$. While not supported with data, preoperative chemotherapy appears ethically acceptable given that it is currently the subject of a randomized controlled trial ${ }^{89}$ and has been introduced as routine in some centres ${ }^{90}$.

\section{Clinical Risk Tools}

Clinical tools for GBC detected incidentally after cholecystectomy or diagnosed on imaging are few, were developed on small cohorts, are not well validated, and include information available only after surgery ${ }^{91-93}$. One scoring system focused on preoperative factors, but included both surgical and nonsurgical (metastatic) cases $^{94}$. That system uses age, Eastern Cooperative Oncology Group performance status, serum alkaline phosphatase, tumour size, and presence of metastasis to create a score from 0 to 30 to predict Os. In surgical cases, median survival is 37 months for scores of 0-21.9, 30 months for scores of 22-25.9, 15 months for scores of 26-29.9, and 8 months for a score of 30 .

\section{Intrahepatic Cholangiocarcinoma}

\section{Hepatectomy}

The typical curative-intent management of ICC is hepatectomy, with a 5 -year os of $30 \%{ }^{95}$. Postoperative mortality of $7.6 \%$ after hepatectomy for primary liver tumours is considerably higher than the rate typically cited for all hepatectomies ${ }^{19}$.

\section{Systemic Therapy}

Retrospective cohort studies have reported on neoadjuvant chemotherapy for initially unresectable ICC. Of 74 patients with locally advanced unresectable disease receiving various chemotherapy regimens $(59 \%$ being doublet gemcitabine-oxaliplatin), 39 were converted to resectability (53\%) after a median of 6 cycles $^{96}$. Median os after resection in that group was 24 months, compared with 26 months in initially resectable ICC.

\section{Ablation}

Ablative therapies appear to be effective in ICC, although experience is limited. A meta-analysis of RFA reported local tumour progression in $21 \%$ of patients and a 5 -year Os of $25 \%{ }^{97}$. A small series of SBRT observed a median os of 22 months with a 1-year local control rate of $78 \%{ }^{98}$.

\section{TACE}

Radioembolization with ${ }^{90} \mathrm{Y}$ and TACE have both been used for unresectable ICC. Median os was similar for the combination of drug-eluting beads containing irinotecan plus chemotherapy and for chemotherapy 
(gemcitabine-oxaliplatin) alone, at 12 and 11 months respectively; median os with conventional TACE was 6 months ${ }^{99}$. In a meta-analysis, ${ }^{90} \mathrm{Y}$ radioembolization was associated with a median os of 15 months ${ }^{100}$.

\section{Clinical Risk Tools}

Various prognostic tools have been described for ICC, but none are universally accepted ${ }^{101}$. Few tools incorporate patient characteristics such as age and sex, and most rely on pathology variables that might not be available before surgery $^{102-104}$. Recently, the LabScore combined carbohydrate antigen 19-9, neutrophil-to-lymphocyte ratio, platelets, and albumin into a published formula to predict 5 -year os after resection as $55 \%$ for a score of $0-9,38 \%$ for a score of $10-19$, and $22 \%$ for a score of 20 or more ${ }^{105}$.

\section{Hilar Cholangiocarcinoma Hepatectomy and Oncologic Outcomes}

For the few resectable hilar cholangiocarcinomas that are amenable to curative-intent treatment, the 5-year os is $8.4 \%$ in node-positive disease and $25.9 \%$ in node-negative disease $^{106}$. Given the extent and complexity of hepatectomy, and the need for a biliary-enteric anastomosis, major morbidity and mortality are higher in these patients ${ }^{107}$.

\section{Chemoradiation}

Conversion from unresectable to resectable hilar cholangiocarcinoma has been reported with chemoradiation (11 of 15 patients at a single institution) ${ }^{108}$. For resectable or borderline resectable disease, single-centre experiences with neoadjuvant chemoradiation for mixed cohorts of hilar and extrahepatic cholangiocarcinoma have reported $90 \%-100 \%$ R0 resection rates and $25 \%-33 \%$ complete pathologic responses ${ }^{109,110}$. Thus, tumour control appears achievable with preoperative chemoradiation if surgery has to be delayed. Finally, for the select few patients with technically unresectable hilar cholangiocarcinoma who might be considered for transplantation, neoadjuvant chemoradiation offers benefits in selection and prognosis ${ }^{111}$.

\section{SUMMARY}

The COVID-19 pandemic affects many treatment decisions for patients with and without the virus. Reduced access to the operating room and lessened availability of critical care services for postoperative management could force the use of treatment strategies outside the standard of care to delay surgery. A variety of delaying strategies could be considered if operating room availability decreases. All strategies have pros and cons for patients and health systems that should be weighed. The quality and availability of data are variable. Evidence comparing typical curativeintent surgery with delaying options is rarely available. In the present review, we summarized the available data to provide some guidance with respect to possible delaying strategies depending on patient, resource, institution, and systems factors. Multidisciplinary team discussions should be leveraged to consider patient- and tumour-specific information to make the best possible decision for each individual case. Finally, it is important to emphasize that the nonsurgical therapies discussed should be used only with the goal of maintaining tumour control while awaiting hepatectomy. Curative-intent resection remains the cornerstone of therapy and the ultimate goal.

\section{CONFLICT OF INTEREST DISCLOSURES}

We have read and understood Current Oncology's policy on disclosing conflicts of interest, and we declare that we have none.

\section{AUTHOR AFFILIATIONS}

*Canada: Department of Surgery, University of Toronto, Toronto, ON (Bennett, Callegaro, Hallet); Department of Radiation Oncology, University of Toronto, Toronto, ON (Myrehaug); Odette Cancer Centre, Sunnybrook Health Sciences Centre, Toronto, ON (Hallet); 'Norway: Department of Gastrointestinal Surgery, Stavanger University Hospital, Stavanger, and Department of Clinical Medicine, University of Bergen, Bergen; ${ }^{\ddagger}$ United States: Division of Surgical Oncology, Department of Surgery, University of California, Davis, CA (Gholami); Virginia Mason Cancer Institute, Seattle, WA (Kennecke); ${ }^{\circledR}$ France: Department of Surgery, Institut Hospitalo-Universitaire de Strasbourg, Strasbourg; "Philippines: Institute of Surgery, St. Luke's Medical Center, Quezon City; Department of Surgery, Makati Medical Center, Makati; and Department of General Surgery, National Kidney and Transplant

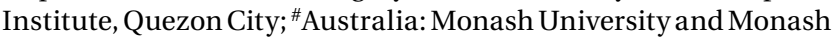
Health, Melbourne; **Belgium: Department of Oncology, University Hospital Antwerp, Antwerp; ${ }^{\dagger}$ Italy: Department of Surgery, Fondazione IRCCS Istituto Nazionale Tumori, Milan.

\section{REFERENCES}

1. Spinelli A, Pellino G. Covid-19 pandemic: perspectives on an unfolding crisis. Br J Surg 2020;107:785-7.

2. Ueda M, Martins R, Hendrie PC, et al. Managing cancer care during the COVID-19 pandemic: agility and collaboration toward a common goal. J Natl Compr Canc Netw 2020;: [online ahead of print].

3. Brindle ME, Gawande A. Managing CoviD-19 in surgical systems. Ann Surg 2020;272:e1-2.

4. Iacobucci G. COVID-19: all non-urgent elective surgery is suspended for at least three months in England. BMJ 2020; 368:m1106.

5. American College of Surgeons (ACS). COVID-19: Elective Case Triage Guidelines for Surgical Care [Web page]. Chicago, IL: ACS; 2020. [Available at: https://www.facs.org/covid-19/ clinical-guidance/elective-case; cited 27 August 2020]

6. Morrison S. Elective surgery [media release]. Canberra, Australia; Government of Australia; 2020. [Available online at: https://www.pm.gov.au/media/elective-surgery; cited 25 March 2020]

7. National Comprehensive Cancer Network (NCCN). Short-Term Recommendations for Cutaneous Melanoma Management During COVID-19 Pandemic. Plymouth Meeting, PA: NCCN; 2020. [Available online at: https://www.nccn.org/covid-19/ pdf/Melanoma.pdf; cited 27 August 2020]

8. Dietz JR, Moran MS, Isakoff SJ, et al. Recommendations for prioritization, treatment, and triage of breast cancer patients during the COVID-19 pandemic. The COVID-19 Pandemic Breast Cancer Consortium. Breast Cancer Res Treat 2020;181:487-97.

9. Bartlett DL, Howe JR, Chang G, et al. Management of cancer surgery cases during the COVID-19 pandemic: considerations. Ann Surg Oncol 2020;27:1717-20.

10. Brat GA, Hersey SP, Chhabra K, Gupta A, Scott J. Protecting surgical teams during the COVID-19 outbreak: a narrative review and clinical considerations. Ann Surg 2020;:[online ahead of print]. 
11. Ti LK, Ang LS, Foong TW, Ng BSW. What we do when a CovID-19 patient needs an operation: operating room preparation and guidance. Can J Anaesth 2020;67:756-8.

12. Liang W, Guan W, Chen R, et al. Cancer patients in SARS-Cov-2 infection: a nationwide analysis in China. Lancet Oncol 2020;21:335-7.

13. Guan WJ, Ni ZY, HuY, et al. Clinical characteristics of coronavirus disease 2019 in China. N Engl J Med 2020;382:1708-20.

14. Wang T, Du Z, Zhu F, et al. Comorbidities and multi-organ injuries in the treatment of CovID-19. Lancet 2020;395:e52.

15. Wu Z, McGoogan JM. Characteristics of and important lessons from the coronavirus disease 2019 (COvID-19) outbreak in China: summary of a report of 72314 cases from the Chinese Center for Disease Control and Prevention. JAMA 2020;:[online ahead of print].

16. Dai M, Liu D, Liu M, et al. Patients with cancer appear more vulnerable to SARS-COV-2: a multi-center study during the COVID-19 outbreak. Cancer Discov 2020;:[online ahead of print].

17. Lei S, Jiang F, Su W, et al. Clinical characteristics and outcomes of patients undergoing surgeries during the incubation period of COVID-19 infection. eClinicalMedicine 2020;:[online ahead of print].

18. COVIDSurg Collaborative. Mortality and pulmonary complications in patients undergoing surgery with perioperative SARS-COV-2 infection: an international cohort study. Lancet 2020;396:27-38.

19. Lassen K, Nymo LS, Olsen F, et al. Contemporary practice and short-term outcomes after liver resections in a complete national cohort. Langenbecks Arch Surg 2019;404:11-19.

20. Bennett S, Baker LK, Martel G, et al. The impact of perioperative red blood cell transfusions in patients undergoing liver resection: a systematic review. HPB (Oxford) 2017;19:321-30.

21. Ulyett S, Shahtahmassebi G, Aroori S, et al. Comparison of risk-scoring systems in the prediction of outcome after liver resection. Perioper Med (Lond) 2017;6:22.

22. Kim SH, Lee JG, Kwon SY, Lim JH, Kim WO, Kim KS. Is close monitoring in the intensive care unit necessary after elective liver resection? J Korean Surg Soc 2012;83:155-61.

23. Cohen ME, Bilimoria KY, Ko CY, Hall BL. Development of an American College of Surgeons National Surgery Quality Improvement Program: morbidity and mortality risk calculator for colorectal surgery. J Am Coll Surg 2009;208:1009-16.

24. Lemke M, Law $\mathrm{CH}, \mathrm{Li} \mathrm{J}$, et al. on behalf of the HPB CONCEPT team. Three-point transfusion risk score in hepatectomy. Br J Surg 2017;104:434-42.

25. Lemke M, Mahar A, Karanicolas PJ, Coburn NG, Law CHL, Hallet J. Three point transfusion risk score in hepatectomy: an external validation using the American College of Surgeons-National Surgical Quality Improvement Program (ACS-NSQIP). HPB (Oxford) 2018;20:669-75.

26. Pagano MB, Hess JR, Tsang HC, et al. Prepare to adapt: blood supply and transfusion support during the first 2 weeks of the 2019 novel coronavirus (COVID-19) pandemic affecting Washington State. Transfusion 2020;60:908-11.

27. Al-Shamsi HO, Alhazzani W, Alhuraiji A, et al. A practical approach to the management of cancer patients during the novel coronavirus disease 2019 (COVID-19) pandemic: an international collaborative group. Oncologist 2020;25:e936-45.

28. Lahat E, Eshkenazy R, Zendel A, et al. Complications after percutaneous ablation of liver tumors: a systematic review. Hepatobiliary Surg Nutr 2014;3:317-23.

29. Sanuki N, Takeda A, Oku Y, et al. Stereotactic body radiotherapy for small hepatocellular carcinoma: a retrospective outcome analysis in 185 patients. Acta Oncol2014;53:399-404.

30. Takeda A, Sanuki N, Kunieda E. Role of stereotactic body radiotherapy for oligometastasis from colorectal cancer. World J Gastroenterol 2014;20:4220-9.
31. Cheng X, Sun P, Hu QG, Song ZF, Xiong J, Zheng QC. Transarterial (chemo) embolization for curative resection of hepatocellular carcinoma: a systematic review and meta-analyses. J Cancer Res Clin Oncol 2014;140:1159-70.

32. Martin RCG, Joshi J, Robbins K, et al. Hepatic intra-arterial injection of drug-eluting bead, irinotecan (DEBIRI) in unresectable colorectal liver metastases refractory to systemic chemotherapy: results of multi-institutional study. Ann Surg Oncol 2011;18:192-8.

33. Fiorentini G, Aliberti C, Mulazzani L, et al. Chemoembolization in colorectal liver metastases: the rebirth. Anticancer Res 2014;34:575-84.

34. Hallet J, Sa Cunha A, Adam R, et al. on behalf of the French Colorectal Liver Metastases Working Group, Association Française de Chirurgie. Factors influencing recurrence following initial hepatectomy for colorectal liver metastases. Br J Surg 2016;103:1366-76.

35. Pawlik TM, Scoggins CR, Zorzi D, et al. Effect of surgical margin status on survival and site of recurrence after hepatic resection for colorectal metastases. Ann Surg 2005;241:715-22.

36. Leal JN, Bressan AK, Vachharajani N, et al. Time-to-surgery and survival outcomes in resectable colorectal liver metastases: a multi-institutional evaluation. J Am Coll Surg 2016; 222:766-79.

37. Folprecht G, Gruenberger T, Bechstein W, et al. Survival of patients with initially unresectable colorectal liver metastases treated with FOLFOX/cetuximab or FOLFIRI/cetuximab in a multidisciplinary concept (CELIM study). Ann Oncol 2014;25:1018-25.

38. Ruers T, Van Coevorden F, Punt CJ, et al. on behalf of the European Organisation for Research and Treatment of Cancer, the Gastro-Intestinal Tract Cancer Group, the Arbeitsgruppe Lebermetastasen und tumoren in der Chirurgischen Arbeitsgemeinschaft Onkologie, and the National Cancer Research Institute Colorectal Clinical Study Group. Local treatment of unresectable colorectal liver metastases: results of a randomized phase II trial. J Natl Cancer Inst 2017;109:djx015.

39. Ciliberto D, Prati U, Roveda L, et al. Role of systemic chemotherapy in the management of resected or resectable colorectal liver metastases: a systematic review and meta-analysis of randomized controlled trials. Oncol Rep 2012;27:1849-56.

40. Nordlinger B, Sorbye H, Glimelius B, et al. Perioperative FOLFOX4 chemotherapy and surgery versus surgery alone for resectable liver metastases from colorectal cancer (EORTC 40983): long-term results of a randomised, controlled, phase 3 trial. Lancet Oncol 2013;14:1208-15.

41. American College of Surgeons (ACS). COVID-19: Guidance for Triage of Non-Emergent Surgical Procedures [Web page] Chicago, IL: ACS; 2020. [Available at: https://www.facs.org/ covid-19/clinical-guidance/triage; cited 28 August 2020]

42. Aloia TA, Vauthey JN, Loyer EM, et al. Solitary colorectal liver metastasis: resection determines outcome. Arch Surg 2006;141:460-6.

43. White RR, Avital I, Sofocleous CT, et al. Rates and patterns of recurrence for percutaneous radiofrequency ablation and open wedge resection for solitary colorectal liver metastasis. J Gastrointest Surg 2007;11:256-63.

44. Schiffman SC, Bower M, Brown RE, Martin RCG, McMasters $\mathrm{KM}$, Scoggins CR. Hepatectomy is superior to thermal ablation for patients with a solitary colorectal liver metastasis. J Gastrointest Surg 2010;14:1881-6.

45. Puijk RS, Ruarus AH, Vroomen LGPH, et al. Colorectal liver metastases: surgery versus thermal ablation (COLLISION) - a phase III single-blind prospective randomized controlled trial. BMC Cancer 2018;18:821.

46. Wild AT, Yamada Y. Treatment options in oligometastatic disease: stereotactic body radiation therapy-focus on colorectal cancer. Visc Med 2017;33:54-61. 
47. Gasent Blesa JM, Laforga Canales J, Alberola Soler A, Peiró Monzó F, Bertelli Puche J, Alberola Candel V. Isolated colorectal liver metastases locally progressing after stereotactic body radiotherapy rescued with surgery. Curr Oncol 2009;16:76-80.

48. Fiorentini G, Aliberti C, Tilli M, et al. Intra-arterial infusion of irinotecan-loaded drug-eluting beads (DEBIRI) versus intravenous therapy (FOLFIRI) for hepatic metastases from colorectal cancer: final results of a phase III study. Anticancer Res 2012;32:1387-95.

49. Mahar AL, Compton C, Halabi S, Hess KR, Weiser MR, Groome PA. Personalizing prognosis in colorectal cancer: a systematic review of the quality and nature of clinical prognostic tools for survival outcomes. J Surg Oncol 2017;116:969-82.

50. Fong Y, Fortner J, Sun RL, Brennan MF, Blumgart LH. Clinical score for predicting recurrence after hepatic resection for metastatic colorectal cancer: analysis of 1001 consecutive cases. Ann Surg 1999;230:309-18.

51. Beamish P, Lemke M, Li J, et al. on behalf of the HPB CONCEPT team. Validation of Clinical Risk Score for colorectal liver metastases resected in a contemporary multicenter cohort. HPB (Oxford) 2017;19:675-81.

52. Margonis GA, Sasaki K, Gholami S, et al. Genetic and Morphological Evaluation (GAME) score for patients with colorectal liver metastases. BrJ Surg 2018;105:1210-20.

53. Forner A, Reig M, Bruix J. Hepatocellular carcinoma. Lancet 2018;391:1301-14.

54. Wada H, Eguchi H, Noda T, et al. Selection criteria for hepatic resection in intermediate-stage (BCLC stage B) multiple hepatocellular carcinoma. Surgery 2016;160:1227-35.

55. Tsilimigras DI, Bagante F, Sahara K, et al. Prognosis after resection of Barcelona Clinic Liver Cancer (BCLC) stage 0, A, and B hepatocellular carcinoma: a comprehensive assessment of the current BCLC classification. Ann Surg Oncol 2019;26:3693-700.

56. Gori A, Dondossola D, Antonelli B, et al. Coronavirus disease 2019 and transplantation: a view from the inside. Am J Transplant 2020;20:1939-40.

57. Kumar D, Manuel O, Natori Y, et al. CoviD-19: a global transplant perspective on successfully navigating a pandemic. Am J Transplant 2020;20:1773-9.

58. Michaels MG, La Hoz RM, Danziger-Isakov L, et al. Coronavirus disease 2019: implications of emerging infections for transplantation. Am J Transplant 2020;:[in press].

59. Livraghi T, Meloni F, Di Stasi M, et al. Sustained complete response and complications rates after radiofrequency ablation of very early hepatocellular carcinoma in cirrhosis: is resection still the treatment of choice? Hepatology 2008;47:82-9.

60. Mulier S, Ni Y, Jamart J, Ruers T, Marchal G, Michel L. Local recurrence after hepatic radiofrequency coagulation: multivariate meta-analysis and review of contributing factors. Ann Surg 2005;242:158-71.

61. Wang Y, Luo Q, Li Y, Deng S, Wei S, Li X. Radiofrequency ablation versus hepatic resection for small hepatocellular carcinomas: a meta-analysis of randomized and nonrandomized controlled trials. PLoS One 2014;9:e84484.

62. Muaddi $\mathrm{H}, \mathrm{Al}$-Adra DP, Beecroft $\mathrm{R}$, et al. Liver transplantation is equally effective as a salvage therapy for patients with hepatocellular carcinoma recurrence following radiofrequency ablation or liver resection with curative intent. Ann Surg Oncol 2018;25:991-9.

63. Cárdenes HR, Price TR, Perkins SM, et al. Phase I feasibility trial of stereotactic body radiation therapy for primary hepatocellular carcinoma. Clin Transl Oncol 2010;12:218-25.

64. Bujold A, Massey CA, Kim JJ, et al. Sequential phase I and II trials of stereotactic body radiotherapy for locally advanced hepatocellular carcinoma. J Clin Oncol 2013;31:1631-9.

65. Lasley FD, Mannina EM, Johnson CS, et al. Treatment variables related to liver toxicity in patients with hepatocellular carcinoma, Child-Pugh class A and B enrolled in a phase 1-2 trial of stereotactic body radiation therapy. Pract Radiat Oncol 2015;5:e443-9.

66. Culleton S, Jiang H, Haddad CR, et al. Outcomes following definitive stereotactic body radiotherapy for patients with Child-Pugh B or C hepatocellular carcinoma. Radiother Oncol 2014;111:412-17.

67. Sapisochin G, Barry A, Doherty M, et al. Stereotactic body radiotherapy vs. TACE or RFA as a bridge to transplant in patients with hepatocellular carcinoma. An intention-to-treat analysis. J Hepatol 2017;67:92-9.

68. Lo CM, Ngan H, Tso WK, et al. Randomized controlled trial of transarterial lipiodol chemoembolization for unresectable hepatocellular carcinoma. Hepatology 2002;35:1164-71.

69. Chua TC, Liauw W, Saxena A, et al. Systematic review of neoadjuvant transarterial chemoembolization for resectable hepatocellular carcinoma. Liver Int 2010;30:166-74.

70. Tang YL, Qi XS, Guo XZ. Hepatic resection after initial transarterial chemoembolization versus transarterial chemoembolization alone for the treatment of hepatocellular carcinoma: a meta-analysis of observational studies. Asian Pac J Cancer Prev 2015;16:7871-4.

71. Kudo M. Lenvatinib may drastically change the treatment landscape of hepatocellular carcinoma. Liver Cancer 2018; 7:1-19.

72. Kudo M, Finn RS, Qin S, et al. Lenvatinib versus sorafenib in first-line treatment of patients with unresectable hepatocellular carcinoma: a randomised phase 3 non-inferiority trial. Lancet 2018;391:1163-73.

73. Yarchoan M, Agarwal P, Villanueva A, et al. Recent developments and therapeutic strategies against hepatocellular carcinoma. Cancer Res 2019;79:4326-30.

74. Dasari BV, Kamarajah SK, Hodson J, et al. Development and validation of a risk score to predict the overall survival following surgical resection of hepatocellular carcinoma in non-cirrhotic liver. HPB (Oxford) 2020;22:383-90.

75. Fong Y, Jarnagin W, Blumgart LH. Gallbladder cancer: comparison of patients presenting initially for definitive operation with those presenting after prior noncurative intervention. Ann Surg 2000;232:557-69.

76. Søreide K, Guest RV, Harrison EM, Kendall TJ, Garden OJ, Wigmore SJ. Systematic review of management of incidental gallbladder cancer after cholecystectomy. Br J Surg 2019;106:32-45.

77. Benson AB, D'Angelica MI, Abbott DE, et al. Guidelines insights: hepatobiliary cancers, version 2.2019. J Natl Compr Canc Netw 2019;17:302-10.

78. Taner CB, Nagorney DM, Donohue JH. Surgical treatment of gallbladder cancer. J Gastrointest Surg 2004;8:83-9.

79. Wagholikar GD, Behari A, Krishnani N, et al. Early gallbladder cancer. J Am Coll Surg 2002;194:137-41.

80. Lee SE, Kim KS, Kim WB, et al. on behalf of the Korean Association of Hepato-Biliary and Pancreas Surgery. Practical guidelines for the surgical treatment of gallbladder cancer. J Korean Med Sci 2014;29:1333-40.

81. Mizuno T, Ebata T, Yokoyama Y, etal. Major hepatectomy with or without pancreatoduodenectomy for advanced gallbladder cancer. Br J Surg 2019;106:626-35.

82. Yamamoto Y, Sugiura T, Ashida R, Okamura Y, Ito T, Uesaka K. Indications for major hepatectomy and combined procedures for advanced gallbladder cancer. BrJ Surg 2017;104:257-66.

83. Lundgren L, Muszynska C, Ros A, et al. Management of incidental gallbladder cancer in a national cohort. Br J Surg 2019;106:1216-27.

84. Kishi Y, Nara S, Esaki M, Hiraoka N, Shimada K. Extent of lymph node dissection in patients with gallbladder cancer. Br J Surg 2018;105:1658-64. 
85. Torén W, Ansari D, Søreide K, Andersson R. Re-resection for incidentally detected gallbladder cancer: weighing in on the options. Eur J Surg Oncol 2020;46:495-7.

86. Søreide K, Harrison EM, Wigmore SJ. Research gaps and unanswered questions in gallbladder cancer. HPB (Oxford) 2018;20:685-6.

87. Primrose JN, Fox RP, Palmer DH, et al. Capecitabine compared with observation in resected biliary tract cancer (BILCAP): a randomised, controlled, multicentre, phase 3 study. Lancet Oncol 2019;20:663-73.

88. Azizi AA, Lamarca A, Valle JW. Systemic therapy of gallbladder cancer: review of first line, maintenance, neoadjuvant and second line therapy specific to gallbladder cancer. Chin Clin Oncol 2019;8:43.

89. Goetze TO, Bechstein WO, Bankstahl US, et al. Neoadjuvant chemotherapy with gemcitabine plus cisplatin followed by radical liver resection versus immediate radical liver resection alone with or without adjuvant chemotherapy in incidentally detected gallbladder carcinoma after simple cholecystectomy or in front of radical resection of BTC (ICC/ ECC) - a phase III study of the German registry of incidental gallbladder carcinoma platform (GR) - the AIO/CALGP/ACOGAIN-trial. BMC Cancer 2020;20:122.

90. Cherkassky L, Jarnagin W. Selecting treatment sequence for patients with incidental gallbladder cancer: a neoadjuvant approach versus upfront surgery. Updates Surg 2019;71:217-25.

91. Bai Y, Liu ZS, Xiong JP, et al. Nomogram to predict overall survival after gallbladder cancer resection in China. World J Gastroenterol 2018;24:5167-78.

92. Chen M, Cao J, Zhang B, Pan L, Cai X. A nomogram for prediction of overall survival in patients with node-negative gallbladder cancer. J Cancer 2019;10:3246-52.

93. Zhang W, Hong HJ, Chen YL. Establishment of a gallbladder cancer-specific survival model to predict prognosis in non-metastatic gallbladder cancer patients after surgical resection. Dig Dis Sci 2018;63:2251-8.

94. Yadav S, Tella SH, Kommalapati A, et al. A novel clinically based staging system for gallbladder cancer. J Natl Compr Canc Netw 2020;18:151-9.

95. Mavros MN, Economopoulos KP, Alexiou VG, Pawlik TM. Treatment and prognosis for patients with intrahepatic cholangiocarcinoma: systematic review and meta-analysis. JAMA Surg 2014;149:565-74.

96. Le Roy B, Gelli M, Pittau G, et al. Neoadjuvant chemotherapy for initially unresectable intrahepatic cholangiocarcinoma. Br J Surg 2018;105:839-47.

97. Han K, Ko HK, Kim KW, Won HJ, Shin YM, Kim PN. Radiofrequency ablation in the treatment of unresectable intrahepatic cholangiocarcinoma: systematic review and meta-analysis. J Vasc Intervent Radiol 2015;26:943-8.

98. Gkika E, Hallauer L, Kirste S, et al. Stereotactic body radiotherapy (SBRT) for locally advanced intrahepatic and extrahepatic cholangiocarcinoma. BMC Cancer 2017;17:781.

99. Kuhlmann JB, Euringer W, Spangenberg HC, et al. Treatment of unresectable cholangiocarcinoma. Eur J Gastroenterol Hepatol 2012;24:437-43.

100. Al-Adra DP, Gill RS, Axford SJ, Shi X, Kneteman N, Liau SS. Treatment of unresectable intrahepatic cholangiocarcinoma with yttrium-90 radioembolization: a systematic review and pooled analysis. Eur J Surg Oncol 2015;41:120-7.

101. Buettner S, Galjart B, van Vugt JLA, et al. Performance of prognostic scores and staging systems in predicting longterm survival outcomes after surgery for intrahepatic cholangiocarcinoma. J Surg Oncol 2017;116:1085-95.

102. Sakamoto Y, Kokudo N, Matsuyama Y, et al. Proposal of a new staging system for intrahepatic cholangiocarcinoma: analysis of surgical patients from a nationwide survey of the Liver Cancer Study Group of Japan. Cancer 2016;122:61-70.

103. Hyder O, Marques H, Pulitano C, et al. A nomogram to predict long-term survival after resection for intrahepatic cholangiocarcinoma: an Eastern and Western experience. JAMA Surg 2014;149:432-8.

104. Wang Y, Li J, Xia Y, et al. Prognostic nomogram for intrahepatic cholangiocarcinoma after partial hepatectomy. J Clin Oncol 2013;31:1188-95.

105. Tsilimigras DI, Mehta R, Aldrighetti L, et al. on behalf of the International Intrahepatic Cholangiocarcinoma Study Group. Development and validation of a laboratory risk score (LabScore) to predict outcomes after resection for intrahepatic cholangiocarcinoma. J Am Coll Surg 2020;230:381-91.

106. Clark CJ, Wood-Wentz CM, Reid-Lombardo KM, Kendrick ML, Huebner M, Que FG. Lymphadenectomy in the staging and treatment of intrahepatic cholangiocarcinoma: a population-based study using the National Cancer Institute SEER database. HPB (Oxford) 2011;13:612-20.

107. Ebata T, Nagino M. Perihilar cholangiocarcinoma with emphasis on presurgical management. In: Jarnagin WM, ed. Blumgart's Surgery of the Liver, Biliary Tract, and Pancreas. 6th ed. Toronto, ON: Elsevier; 2017: 833-85.

108. Sumiyoshi T, Shima Y, Okabayashi T, et al. Chemoradiotherapy for initially unresectable locally advanced cholangiocarcinoma. World J Surg 2018;42:2910-18.

109. McMasters KM, Tuttle TM, Leach SD, et al. Neoadjuvant chemoradiation for extrahepatic cholangiocarcinoma. Am J Surg 1997;174:605-8.

110. Nelson JW, Ghafoori AP, Willett CG, et al. Concurrent chemoradiotherapy in resected extrahepatic cholangiocarcinoma. Int J Radiat Oncol Biol Phys 2009;73:148-53.

111. Cambridge WA, Fairfield C, Powell JJ, et al. Meta-analysis and meta-regression of survival after liver transplantation for unresectable perihilar cholangiocarcinoma. Ann Surg $2020 ;:$ [online ahead of print]. 